\title{
In Memoriam Dr. Oded Bar-Or
}

https://doi.org/10.5628/rpcd.06.01.127

With the death of Oded, the pediatric exercise science community lost one of its founding and most important contributors - and of course, a good friend. Oded's contributions are well documented in his numerous publications and there is no need for reiteration. His insights, questions and research into many domains of childhood and adolescence in the context of physical activity, sport, obesity and temperature regulation, among others, have opened new vistas for the present and subsequent generations of sport scientists. What will be missed is his personal touch - pleasant personality, sly sense of humor, work ethic, kindness, and constant focus on youth, i.e., the next generation.

The unfortunate inevitability of death influences individuals differently. I am no exception. For a good part of our careers, Oded and I participated in many meetings. Given our interests in children and adolescents, we often ended up on the same programs. We were colleagues due to our mutual interests in youth. Over time, collegiality grew into friendship - in other words, colleagues became friends! I believe we reached this point during a one or two hour stroll around the walls of Dubrovnik when we were there for a meeting in 1988. During this stroll - taking in the history of this beautiful walled city - our conversations roamed from church history, architecture, family background, among other topics. I recall Oded asking me where I learned so much about the Catholic Church, specifically the saints. Of course, I referred to my parochial school education in a Polish neighborhood of Brooklyn. In response, Oded noted his own family history in Poland prior to migrating to Israel. We had a lot more in common than ever anticipated. Our friendship blossomed over the years since this fortuitous stroll.

Oded will be missed by many but he will not be forgotten.

\section{Robert M. Malina, PhD, FACSM}




\section{In Memoriam Prof Dr Oded Bar-Or}

It is with the deepest regret that I and my colleagues of the faculty of Kinesiology and Rehabilitation Sciences of the KULeuven learned that our esteemed colleague and dear friend Prof Dr Oded Bar-Or passed away on the evening of December 8, 2005. Of course, a number of us, already knew about his illness but we all have hoped that he would have been among us for a much longer time.

I learned to know Oded through the meetings of the European Paediatric Work Physiology Group. He was an active member of that group and after Prof Dr Joseph Rutenfranz passed away Oded became one of the leaders. Later on, I learned to know Oded better through a number of International Organization in which Dr Bar-Or always played a major and leading role.

I will remember Oded as a dear friend, with a great personality and as one of the best scholars over the last decades in the field of paediatric exercise science. He was a prime mover and leader in many organizations and through his publications and lectures that he delivered worldwide he inspired a great number of young scientists.

It has been my honour and pleasure to work under his guidance and leadership in a number of scientific organizations and for a couple of scientific monographs that he edited.

His substantial impact on paediatric exercise science, his scientific contributions, leadership and personality will long be appreciated and fondly remembered by all those who have known him.

\section{Prof Dr Em Gaston Beunen}

Leuven, January 232006 
Nas últimas imagens do filme O ÚLTIMO SAMURAI, há uma troca de palavras entre o "artista" e o imperador. Pergunta o imperador: diz-me como morreu o meu mestre. A resposta não se fez esperar: prefiro falar-te do modo como viveu.

Este PEDAÇO DE CONVERSA, que é de grande importância acerca do modo como nos referimos aqueles que já não partilham connosco o mundo das formas, mas sim DOS NOMES E DOS SENTIDOS MAIS ÍNTIMOS, é para mim muito importante. Precisamente porque gostaria de escrever algumas linhas, e muito breves, sobre um amigo da nossa Faculdade, um dos maiores investigadores do mundo acerca do exercício em idades pediátricas, uma voz sempre escutada, e com muita atenção, em qualquer lugar - o PROF. ODED BAR-OR.

Guardo com especial carinho a participação num congresso na África do Sul em 1995, por ter tido grandes companheiros nesta aventura (o Manuel António, o António Prista e o Leonardo Nhantumbo). Entre investigadores prestigiados de várias partes do globo, contava-se o Prof. Oded BarOr. Numa das noites, em plena savana, e junto a uma fogueira que não se extinguiu, tive a honra de conversar longamente com o Prof. Oded Bar-Or sobre a sua e a minha vida, acerca da sua formação enquanto médico em Israel, e como investigador nos EUA e Canadá. Falou-me de muita coisa, sobretudo do modo como se deve estar na vida académica, das várias maneiras como deveríamos responder aos desafios que nos colocam, mas acima de tudo acerca da atitude a ter com a investigação com crianças e jovens. Das etapas que tal atitude consignam, sobretudo da utilidade do trabalho, uma espécie de "apostolado" à volta das crianças. Sempre ao seu serviço, sempre uma voz a defender os seus interesses. Sempre presente e disponível.

Também em 1995 tive a honra de participar na organização, na FCDEF, de um seminário internacional que contou com a presença de vários amigos da nossa escola: os Profs. Gaston Beunen, Albrecht Claessens, Martine Thomis, António Prista, Sieuve Monteiro e Jorge Sequeiros. Também estava presente o Prof. Oded Bar-Or. Foi a primeira vez que veio ao Porto. Rapidamente se enamorou das suas gentes e desta cidade. Bem mais importante foi a sua disponibilidade em estar ao nosso lado. E sempre assim foi. Esteve connosco cerca de uma semana. Mais uma vez tive a honra de o acompanhar, de partilhar a sua presença, a sua atenção e interesse relativamente a tudo que se fazia na FCDEF. Sobre os seus docentes, alguns dos quais se tornariam, também, seus amigos. Em 2003 realizamos no Porto o Pediatric Work Physiology honrando a sua presença. Uma festa em torno do seu nome. Com a presença de alguns dos mais destacados investigadores do mundo e seus amigos (Professores Claude Bouchard, Robert Malina, Gaston Beunen, Cameron Blimkie, Thomas Rowland, entre outros). Nessa semana de Setembro vivemos numa festa contínua de ciência e de grande amizade. De descoberta e de exposição da amizade forte dos nortenhos e dos amigos da FCDEF. Prestamos-lhe, à nossa maneira, uma homenagem. O Prof. Oded Bar-Or passou a ser da nossa família. Ficou encantado, comovido. Penso que o seu coração, e o da sua esposa, passaram a ter um espaço portuense. Que não conseguiram apagar. Em 2005 recebi a notícia que já não se encontrava mais entre aqueles que convencionalmente designamos por "mundo dos vivos". Não é verdade. Se o 
Prof. Oded Bar-Or fosse exclusivamente o seu corpo físico tal seria verdade. Mas não é. Este querido amigo e Professor está também na nossa escola sempre que nos lembrarmos dele, sempre que invocarmos o seu nome, sempre que tivermos à mão um dos seus escritos, sempre que pensarmos em crianças e exercício, sempre que pensarmos neste querido amigo que também faz parte do "corpo docente" da FCDEF.

Gosto muito de um livro de poesia do grande poeta sul-americano Amado Nervo, cujo título é precisamente "Plenitude". Nele escreve acerca da morte. Alguns dos seus versos rezam assim:

Choras os teus mortos

Com tanta intensidade

Que até parece que és eterno.

\section{Não morreram!}

Partiram antes.

Deixa que ao menos

Repousem nos verdes campos da Paz, Os seus pés de tanto andarem.

Não sei se conseguiremos preencher o seu espaço, tal era a sua grandeza. Fazes-nos muita falta, querido amigo. Mas também sei, que o facto inelutável desta "ausência" nos torna mais vigilantes, disponíveis e responsáveis para segurarmos o facho que firmemente tiveste na mão.

José António Ribeiro Maia

Faculdade de Desporto da Universidade do Porto Portugal 